\title{
Preparation and characterization of nanoparticles formed through stereocomplexation between enantiomeric poly( $\gamma$-glutamic acid)-graft-poly(lactide) copolymers
}

\author{
Ye Zhu' ${ }^{1}$, Takami Akagi ${ }^{1,2}$ and Mitsuru Akashi ${ }^{1,2}$ \\ Novel amphiphilic graft copolymers composed of poly $(\gamma$-glutamic acid) $(\gamma-P G A)$ as a hydrophilic backbone and enantiomeric \\ poly(lactide) (PLA) as hydrophobic side chains were successfully synthesized by using 1-ethyl-3-(3-dimethylaminopropyl)- \\ carbodiimide (EDC) as the coupling agent. The number of poly(L-lactide) (PLLA) or poly(D-lactide) (PDLA) chains grafted onto \\ the $\gamma$-PGA main chain was enhanced by increasing the feed ratio of PLA to $\gamma$-PGA in the reaction system. Nanoparticles (NPs) \\ self-assembled from $\gamma$-PGA-graft-PLLA, $\gamma$-PGA-graft-PDLA and equal molar mixtures of enantiomeric $\gamma$-PGA-graft-PLLA and \\ $\gamma$-PGA-graft-PDLA copolymers were formed in aqueous solution. The mean diameters of the NPs formed by equal molar \\ mixtures of enantiomeric $\gamma$-PGA-graft-PLLA and $\gamma$-PGA-graft-PDLA copolymers (stereocomplex NPs) were in the range from 150 \\ to $250 \mathrm{~nm}$, and these NPs with reactive functional groups would have great potential to be used as stable delivery vehicles for \\ pharmaceutical and biomedical applications.
}

Polymer Journal (2013) 45, 560-566; doi:10.1038/pj.2012.174; published online 26 September 2012

Keywords: amphiphilic copolymers; nanoparticles; poly(lactide); poly( $\gamma$-glutamic acid); stereocomplexation

\section{INTRODUCTION}

Nanostructures prepared from the self-assembly of amphiphilic block or graft copolymers in aqueous solution have attracted widespread attention in the biomedical and pharmaceutical fields. ${ }^{1-4}$ In particular, spherical micelle-like nanoparticles (NPs) composed of hydrophobic inner cores and hydrophilic shells have been extensively studied over the past decade as delivery vehicles for chemical therapies. ${ }^{5-9}$ As a delivery vehicle, the one remaining challenge for particle-based delivery systems is the inferior in vivo stability. ${ }^{10}$ Normally, chemical cross-linking is adapted to improve NPs stability, but the use of cross-linkers may unfavorably affect the bioactivity of the encapsulated cargo and the biodegradability of the delivery system. ${ }^{11}$ Therefore, stabilization of the particle core through physical interactions such as stereocomplexation is an alternative strategy.

Typical examples of polymers used for stereocomplexes are poly (methyl methacrylate) ${ }^{12}$ and PLA. ${ }^{13}$ Stereocomplexation between the L and D type enantiomers of PLA are the most commonly investigated, not only owing to their biodegradability and biocompatibility but also because they are hydrolyzable in the human body, as well as in each environment. The hydrolytic degradation of PLA is affected by various different factors such as the chemical configuration, molecular weight, crystallinity and environmental conditions. This suggests the possibility of tuning the degradation rate over a wide range of homopolymer poly(L-lactides) (PLLAs), poly(D-lactides) (PDLAs) and stereocomplexed PLA composites. In the previous studies, PLLA/PDLA (PLLA/PDLA)-containing copolymers of various architectures were synthesized to form stereocomplex selfassemblies. ${ }^{14-18}$ Leroux et al. ${ }^{19}$ reported that the block copolymer stereocomplex micelles obtained from mixtures of poly(ethylene glycol)-b-PLLA (PEG-PLLA) and PEG-PDLA exhibited stronger thermodynamic properties than micelles formed by isotactic copolymers alone. However, the majority of the researches performed on the formation of NPs through stereocomplexation between PLA enantiomers are based on polymers without reactive functional groups as a side chain, while preparation of stereocomplex NPs through biodegradable copolymer systems and their applications as delivery system were seldom reported. ${ }^{20}$

Poly $(\gamma$-glutamic acid) ( $\gamma$-PGA), a naturally occurring water-soluble poly (amino acid), has been considered to possess the unique properties of biodegradability, immunogenicity and immunoreactivity. ${ }^{21,22}$ In a previous study from our group, size-controllable NPs that can be used as an effective vaccine delivery system were prepared using a hydrophobic amino acid (L-phenylalanine ethylester, Phe) modified

${ }^{1}$ Department of Applied Chemistry, Graduate School of Engineering, Osaka University, 2-1 Yamada-oka, Suita, Osaka, Japan and 2Japan Science and Technology Agent (JST), Core Research for Evolutional Science and Technology (CREST), Saitama, Japan

Correspondence: Dr M Akashi, Department of Applied Chemistry, Graduate School of Engineering, Osaka University, 2-1 Yamada-oka, Suita, Osaka 565-0871, Japan. E-mail: akashi@chem.eng.osaka-u.ac.jp

Received 15 June 2012; revised 15 August 2012; accepted 18 August 2012; published online 26 September 2012 
$\gamma$-PGA-graft-Phe ( $\gamma$-PGA-Phe) copolymer. ${ }^{23-27}$ NPs composed of amphiphilic $\gamma$-PGA-Phe with a $>50 \%$ grafting degree of Phe could covalently immobilize proteins and peptides via carboxyl groups located on the NP surfaces. ${ }^{28,29}$ However, the $\gamma$-PGA-Phe NPs still have some problems for in vivo applications, such as a relatively low drug-loading efficiency on the surfaces and difficulty in controlling the degradation process. In order to improve these drawbacks, other types of hydrophobic groups or biodegradable hydrophobic polymers, such as PLA, should be employed as the hydrophobic part to modify the $\gamma$-PGA main chains through the functional carboxyl groups. $\gamma$-PGA contains multiple carboxyl groups, and have therefore found utility as hydrophilic and bioactive segments in some hybrid biomaterials. One promising approach to regulate the hydrophobicity of $\gamma$-PGA with low degree of substitution is the introduction of hydrophobic polymers. It is considered that the hydrophilic-hydrophobic balance can be easily modulated as a function of the degree of substitution (grafting degree) and the degree of polymerization. The graft copolymer structure may provide integration of considerable functionality onto the polymer backbone that can be addressed chemically after the assembly process. In this study, we developed a method to modify $\gamma$-PGA by hydrophobic PLA homopolymers and the characterization of comb-like amphiphilic $\gamma$-PGA-graft-PLLAs ( $\gamma$-PGA- $g$ PLLA) and $\gamma$-PGA-g-PDLAs were investigated. The capability of forming polymer NPs in aqueous solution and the ability of stereocomplex formation between $\gamma$-PGA- $g$-PLLA and $\gamma$-PGA- $g$-PDLA were discussed.

\section{EXPERIMENTAL PROCEDURE}

\section{Materials and methods}

L-lactide and D-lactide were purchased from Purac Biochem BV (Gorinchem, The Netherlands) and recrystallized from ethyl acetate before use. Poly $(\gamma$ glutamic acid) $\left(\gamma\right.$-PGA, $\left.M_{\mathrm{w}}=480000\right)$, decanol $\left(\mathrm{C}_{10} \mathrm{H}_{21} \mathrm{OH}\right)$, stannous octanoate $\left(\mathrm{Sn}(\mathrm{Oct})_{2}\right)$, dehydrated dimethyl sulfoxide (DMSO), 1,4-dioxane, tetrahydrofuran (THF), dimethyl formamide and chloroform were purchased from Wako Pure Chemical Industries (Osaka, Japan), and used without further purification. 1-Ethyl-3-(3-dimethylaminopropyl)-carbodiimide (EDC) and 4 -( $N, N$-dimethylamino)pyridine were purchased from Sigma (St Louis, MO, USA), and were used as received.

\section{Characterization methods}

${ }^{1} \mathrm{H}$ NMR spectra were recorded with a Varian INOVA 600 instrument (Varian Inc., Palo Alto, CA, USA). Gel permeation chromatography measurements for PLLA and PDLA were measured by a JASCO SEC system (JASCO PU-2080, JASCO RI-2031, and JASCO CO-2065, Jasco Crop., Tokyo, Japan) at $40{ }^{\circ} \mathrm{C}$ with a TSKgel $\mathrm{GMH}_{\mathrm{XL}}$ column using chloroform as the eluent at a rate of $0.6 \mathrm{ml} \mathrm{min}{ }^{-1}$. Poly (methyl methacrylate) was used for calibration. The infrared spectra were recorded on a Perkin-Elmer Spectrum 100 (Perkin-Elmer, Yokohama, Japan). The particle sizes of the $\gamma$-PGA- $g$-PLA NPs in aqueous solutions were measured by dynamic light scattering (DLS) using a Zetasizer Nano ZS (Malvern Instruments, UK) at a wavelength of $633 \mathrm{~nm}$ and a detection angle of $173^{\circ}$. The morphology of $\gamma$-PGA- $g$-PLA NPs were observed by scanning electron microscopy (SEM) (JSM-6700F, JEOL) at $5 \mathrm{kV}$. A drop of the NPs suspension was placed on a glass surface, which was fixed on metallic supports with carbon tape. After drying, the samples were coated with osmic acid. The wide-angle X-ray diffraction (XRD) patterns were recorded on a RIGAKU RINT2000 instrument with a $\mathrm{Cu} \mathrm{K} \alpha(\lambda=0.154 \mathrm{~nm})$ source at room temperature, and was operated at $40 \mathrm{kV}$ and $200 \mathrm{~mA}$ with a Ni filter.

\section{Ring opening polymerization of PLA}

Monomeric $\mathrm{L}$ or D-lactide $(5 \mathrm{~g})$, the initiator $\mathrm{C}_{10} \mathrm{H}_{21} \mathrm{OH}(2.6 \mathrm{~g})$ and the catalyst $\mathrm{Sn}(\mathrm{Oct})_{2}(15 \mathrm{mg})$ were sealed into a $300-\mathrm{ml}$ three-neck flask, followed by three vacuum-nitrogen cycles. The reaction flask was then placed on a preheated oil bath at $160^{\circ} \mathrm{C}$. After $6 \mathrm{~h}$, the reaction mixture was cooled to room temperature, dissolved in $\mathrm{CHCl}_{3}$ and precipitated into excess diethyl ether.
The final products were dried under vacuum at $40^{\circ} \mathrm{C}$ overnight. The chemical structure and molecular weight of the PLLA were checked by $600 \mathrm{~Hz}{ }^{1} \mathrm{H}$ NMR and gel permeation chromatography, respectively.

\section{Synthesis of $\gamma$-PGA- $g$-PLA}

Briefly, the $\gamma$-PGA-g-PLLA and $\gamma$-PGA-g-PDLA graft copolymers were synthesized by a coupling reaction between $\gamma$-PGA and PLAs under a nitrogen atmosphere. $\gamma$-PGA $(100 \mathrm{mg}, 0.775$ unit $\mathrm{mmol}$ was dissolved in $10 \mathrm{ml}$ of dehydrated DMSO, and then the stoichiometric amounts of 1-ethyl-3-(3-dimethylaminopropyl)-carbodiimide (149 mg, $0.775 \mathrm{mmol}$ ) and 1-ethyl-3-(3-dimethylaminopropyl)-carbodiimide (EDC) and 4-(N,N-dimethylamino)pyridine $(95 \mathrm{mg}$, $0.775 \mathrm{mmol}$ ) were added to the solution. After all the chemicals were dissolved, $2 \mathrm{ml}$ of pre-dissolved PLLA or PDLA dioxane solution at various concentrations $\left(25,50\right.$ or $\left.100 \mathrm{mg} \mathrm{ml}^{-1}\right)$ was added into the $\gamma$-PGA solution, and the reaction was allowed to continue for $24 \mathrm{~h}$ at room temperature. Next, the reaction mixture was dialyzed with a membrane (molecular weight cutoff of $50000 \mathrm{Da}$ ) against pure water for 2 days, and lyophilized until dry. The obtained products were washed twice by an excess amount of THF to remove the uncoupled PLLA or PDLA. $\gamma$-PGA- $g$-PLLA and $\gamma$-PGA- $g$-PDLA copolymers, which precipitated in THF, were collected by centrifugation and dried under vacuum overnight.

\section{Preparation of polymer NPs}

Polymer NPs were prepared by the dialysis method. $\gamma$-PGA- $g$-PLLA, $\gamma$-PGA- $g$ PDLA and equal molar amounts of PLLA and PDLA copolymer were first dissolved in DMSO, a solvent for both $\gamma$-PGA and PLA, to a concentration of $10 \mathrm{mg} \mathrm{ml}^{-1}$. Then water, which is the non-solvent of PLA, was added dropwise into the copolymer solution with vigorous stirring. When the water content reached 50 vol.\%, the solutions were transferred into a dialysis tube (MWCO: $1000 \mathrm{Da}$ ) for dialysis against pure water for 3 days to remove the DMSO. The particle size and distribution were measured by DLS. The crystallinity of lyophilized particles was characterized by XRD measurements.

\section{RESULTS AND DISCUSSION}

\section{Synthesis of $\gamma$-PGA- $g$-PLA copolymers}

As shown in Scheme 1, $\gamma$-PGA- $g$-PLLA and $\gamma$-PGA- $g$-PDLA copolymers were prepared by a two-step reaction. First, the enantiomeric PLLA $\left(M_{\mathrm{w}}=3000\right)$ and PDLA $\left(M_{\mathrm{w}}=4000\right)$ homopolymers were synthesized by a ring-opening polymerization of the L-lactide or D-lactide monomer with $n$-decanol as the initiator and $\mathrm{Sn}(\mathrm{Oct})_{2}$ as the catalyst. The molecular weight and degree of polymerization were both measured by ${ }^{1} \mathrm{H}$ NMR. Next, graft copolymers with different grafting degree of PLA were synthesized by a coupling reaction between the terminal hydroxyl group in PLA and the carboxylic groups in $\gamma$-PGA $\left(M_{\mathrm{w}}=480000\right)$ using 1-ethyl-3-(3-dimethylaminopropyl)-carbodiimide. After dialysis against water and lyophilization, the copolymers were washed with THF twice to remove the unconjugated PLA. $\gamma$-PGA- $g$-PLA copolymers were precipitated in THF and collected by centrifugation. The characterization of the $\gamma$-PGA- $g$-PLLA and $\gamma$-PGA- $g$-PDLA copolymers thus obtained is summarized in Table 1 . The grafting degree of PLA, the number and hydrophobic content (\%) of the PLAs in the copolymer were calculated from ${ }^{1} \mathrm{H}$ NMR (solvent DMSO- $d_{6}$ ) using the integrals of the methylene peaks (2.20 p.p.m. a- $\mathrm{CH}_{2}$ and 1.74-1.90 p.p.m., b-CH ) in the $\gamma$-PGA backbone and the methine peaks (5.20 p.p.m. e-CH) in the PLA main chain (Figure 1). The conversion degree of PLA was from about 10 to $15 \%$ (Table 1). In this study, terminal secondary hydroxyl group in PLA was used for the formation of ester bond between $\gamma$-PGA and PLA. This is attributed to the fact that the reactivity of secondary hydroxyl group is lower than that of the primary one. However, it is noteworthy that the number of PLA chains per $\gamma$-PGA molecules in the copolymer increased from about 


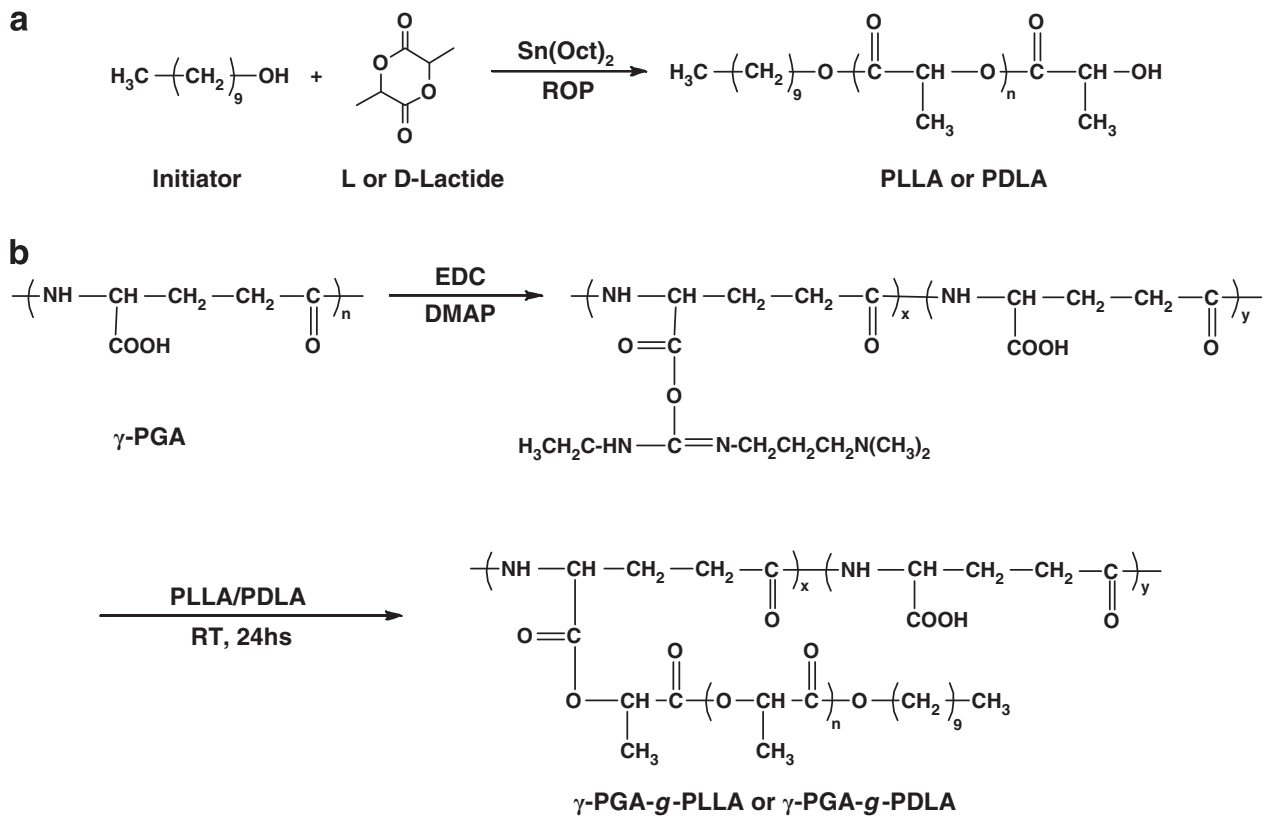

Scheme 1 Synthesis of (a) poly(L-lactide)/poly(D-lactide) (PLLA/PDLA) polymer and (b) poly( $\gamma$-glutamic acid) $(\gamma$-PGA)-g-PLLA/PDLA copolymers.

Table 1 Synthesis of $\gamma$-PGA-g-PLLA and $\gamma$-PGA-g-PDLA copolymers

\begin{tabular}{|c|c|c|c|c|c|c|}
\hline Code & Sample & $\begin{array}{l}\text { Feed } \\
\text { Ratio }^{\mathrm{a}}\end{array}$ & Yield $^{\mathrm{b}}(\%)$ & $\begin{array}{c}\text { Conversion degree } \\
\text { of } P L A^{c}(\%)\end{array}$ & $\begin{array}{l}\text { Number of } \\
\text { grafted PLA }\end{array}$ & $\begin{array}{l}\text { Hydrophobic } \\
\text { Content }^{\text {e }} \% \text { ) }\end{array}$ \\
\hline$\gamma-P G A-L_{1}$ & $\gamma$-PGA-g-PLLA 11 & 0.5 & 53 & 14 & 11 & 7 \\
\hline$\gamma$-PGA-L 3 & $\gamma$-PGA-g-PLLA 22 & 1 & 50 & 14 & 22 & 12 \\
\hline$\gamma$-PGA-D 4 & $\gamma$-PGA-g-PDLA 18 & 1 & 45 & 16 & 18 & 13 \\
\hline$\gamma-P G A-L_{5}$ & $\gamma$-PGA-g-PLLA 37 & 2 & 42 & 12 & 37 & 19 \\
\hline
\end{tabular}

Abbreviations: PGA, poly glutamic acid; PLA, poly(lactide); PLLA, poly(L-lactide).

aFeed ratio $=$ PLA $/ \gamma-P G A(g / g)$.

bYield $=((\gamma-P G A-g-P L A) /(P L A+\gamma-P G A)) \times 100 \%(g / g)$.

${ }^{c}$ Conversion degree of PLA $=(($ Number of PLA chains grafted to $\gamma$-PGA molecule $) /($ feed number of PLA chains per $\gamma$-PGA molecule $)) \times 100 \%$.

${ }^{\mathrm{d}}$ Number of grafted PLLA/PDLA chains was estimated by ${ }^{1} \mathrm{H}$ NMR (solvent DMSO- $d_{6}$ ).

eHydrophobic content was the content of PLA unit in $\%=\left(\left(M_{w}\right.\right.$ of PLA $\times$ zthe number of grafted PLAs $) /\left(M_{w}\right.$ of $\gamma-P G A-g$-PLA copolymers $\left.)\right)$.

7 to 37 by increasing the feed ratio of PLA to $\gamma$-PGA, which demonstrated that the hydrophobic substitution degree of the copolymers can be controlled by altering the amount of PLA feeding into the reaction system.

\section{Solubility of $\boldsymbol{\gamma}$-PGA- $\boldsymbol{g}$-PLA copolymers}

The solubility of $\gamma$-PGA, PLA and the copolymer in water $(\mathrm{pH}=7)$ and several common organic solvents was investigated. $\gamma$-PGA is known to be soluble in water and DMSO, while PLA can be dissolved in THF, chloroform, dimethyl formamide and some other organic solvents (Table 2). However, all of the $\gamma$-PGA- $g$-PLLA and $\gamma$-PGA- $g$ PDLA copolymers with different PLA contents were soluble in DMSO, but could not be dissolved in water or other friendly organic solvents for PLA, such as THF, chloroform and dimethyl formamide. This was probably because of the amphiphilic properties of the copolymer. Although hydrophobic PLAs were connected onto the hydrophilic $\gamma$-PGA and the number of PLA chains grafted onto the copolymers were improved by enhancing the feed molar ratio of PLA, the content of the hydrophobic part in the copolymer is still low, and the majority part of the copolymer consisted of $\gamma$-PGA, which inhibited the dispersion and dissolution of the copolymer in common organic solvents.

\section{Characterization of $\gamma$-PGA- $g$-PLA NPs}

Copolymer- and stereocomplex NPs were prepared by dialysis method, which is a common method to prepare polymer micelles from diblock copolymers. ${ }^{30} \gamma$-PGA- $g$-PLLA, $\gamma$-PGA- $g$-PDLA and equal molar ratios of PLLA and PDLA copolymer were first dissolved in DMSO at a concentration of $10 \mathrm{mg} \mathrm{ml}^{-1}$, and then the solutions were transferred into a dialysis tube (MWCO: $1000 \mathrm{Da}$ ) for dialysis against pure water to form NPs and remove the DMSO (Scheme 2). The size and distribution of NPs with different composition were measured by DLS and listed in Table 3 (mean \pm s.d. of three independent experiments is shown). Owing to their amphiphilic properties, all $\gamma$-PGA- $g$-PLA copolymers with different PLA contents can form nanometer-sized particles in aqueous phase with mean diameters ranging from 35 to $170 \mathrm{~nm}$. This indicated that the size of the NPs increased with the enhancement of hydrophobic 


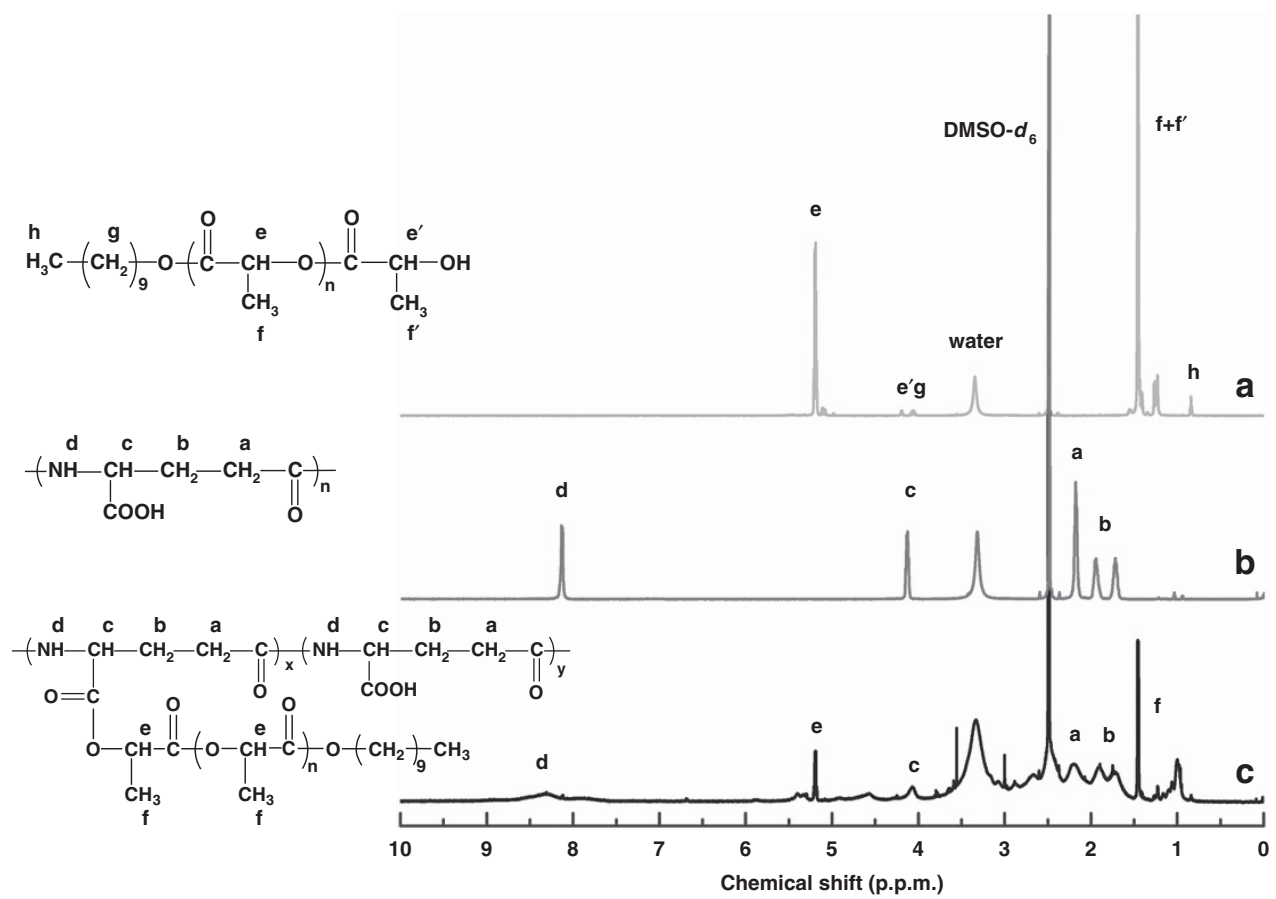

Figure $1{ }^{1}$ H-NMR spectra of (a) poly(lactide) (PLA), (b) poly( $\gamma$-glutamic acid) $(\gamma$-PGA) and (c) $\gamma$-PGA-L5 copolymer. A full color version of this figure is available at Polymer Journal online.

Table 2 Solubility of $\gamma$-PGA-g-PLA copolymers in various solvents

\begin{tabular}{lccccc}
\hline Sample & Water $^{\mathrm{a}}$ & THF & $\mathrm{CHCl}_{3}$ & DMF & DMSO \\
\hline$\gamma$-PGA & + & - & - & - & + \\
PLLA/PDLA & - & + & + & + & + \\
$\gamma$-PGA-L & - & - & - & - & + \\
$\gamma-$ PGA-D $_{2}$ & - & - & - & - & + \\
$\gamma$-PGA-L & - & - & - & - & + \\
$\gamma$-PGA-D & - & - & - & - & + \\
$\gamma$-PGA-L & - & - & - & - & + \\
$\gamma-$ PGA-D $_{6}$ & - & - & - & - & + \\
\hline
\end{tabular}

Abbreviations: $\mathrm{CHCl}_{3}$, chloroform; DMF, dimethyl formamide; DMSO, dehydrated dimethyl sulfoxide; $\gamma$-PGA-g-PLA, poly( $\gamma$-glutamic acid)-graft-poly(L-lactide); PLLA/PDLA, poly(L-lactide)/ poly(D-lactide); THF, 1,4-dioxane, tetrahydrofuran; +, soluble; -, insoluble.

The solubility tests were carried out by ultrasonication for $2 \mathrm{~h}$ at room temperature, at the concentration of $2 \mathrm{mg} \mathrm{ml}^{-1}$.

${ }^{\mathrm{a}} \mathrm{pH}$ of water is 7 .

content in the copolymer. All NPs formed by $\gamma$-PGA- $g$-PLA copolymers showed a single peak in the DLS measurement, except those formed by $\gamma$-PGA- $\mathrm{L}_{1}$ or $\gamma$-PGA- $\mathrm{D}_{2}$, in which relatively large particles with an average diameter of $250 \mathrm{~nm}$ were observed from the DLS measurements. This is probably because the hydrophobic content in $\gamma$-PGA- $\mathrm{L}_{1}$ or $\gamma$-PGA-D $\mathrm{D}_{2}$ copolymers were too low (about $7 \%$ ) to form stable NPs, whereas with the increasing level of PLA content, NPs with more stable structures and narrow distribution were obtained. Thus, the size and distribution of copolymer NPs may be controlled by the number of grafted PLA chains. Compared with NPs formed by $\gamma$-PGA-g-PLLA or $\gamma$-PGA- $g$-PDLA only, the size of stereocomplex NPs formed by an equal molar mixture of PLLA and PDLA copolymers were increasing, whereas the polydispersity factors were slightly decreased for $\gamma$-PGA-L $\mathrm{L}_{5}+\gamma-$ PGA-D $_{6}$ stereocomplex NPs (Figure 2). The increased size implied that, in the case of stereocomplex NP formation, the number of copolymer chains involved into the self-assembling process was increased. The morphology of $\gamma$-PGA-L ${ }_{5}+\gamma$-PGA-D 6 stereocomplex NPs was observed by SEM. As shown in Figure 3, the morphology of stereocomplex NPs prepared from the mixture of $\gamma$-PGA- $\mathrm{L}_{5}$ and $\gamma-\mathrm{PGA}_{-} \mathrm{D}_{6}$ copolymers were spherical with an average diameter of $150 \mathrm{~nm}(n=30)$. The size measured by DLS was higher than that of the SEM method. This difference can be attributed to the difference between the dried and hydrated state. In the case of SEM method, SEM image exhibits the size of sample in dried state, whereas the DLS method involves the measurement of size in the hydrated state. Therefore, in the hydrated state, the NPs will have a higher particle size because of the solvent effect.

To further prove the formation of NPs using $\gamma$-PGA- $g$-PLA, ${ }^{1} \mathrm{H}$ NMR spectroscopy of $\gamma$-PGA- $\mathrm{L}_{3}$ NPs dispersed in DMSO- $d_{6}$ and $\mathrm{D}_{2} \mathrm{O}$ were carried out, as shown in Figure 4. NMR can be employed to study the structure of polymeric NPs, especially for core-shell type particles. As both poly $(\gamma$-glutamic acid $)(\gamma$-PGA) and PLA can dissolve in DMSO- $d_{6}$ and exist in a liquid state, particle formation was not expected. The characteristic peaks of $\gamma$-PGA and PLA are shown in DMSO- $d_{6}$ (Figure 4a). However, as shown in Figure $4 \mathrm{~b}$, the characteristic NMR peaks of PLA (5.20 p.p.m. e-CH and 1.46 p.p.m. $\mathrm{f}-\mathrm{CH}_{3}$ ) disappeared completely using $\mathrm{D}_{2} \mathrm{O}$. These results indicate that the protons of PLA displayed restricted motion, and have a solid-like structure. The NMR peaks of the hydrophilic $\gamma$-PGA chains were detected using $\mathrm{D}_{2} \mathrm{O}$, implying that the structure of NPs consisting of $\gamma$-PGA- $g$-PLA should be a core-shell type composed of inner core of PLA and outer shell of $\gamma$-PGA. However, unlike an amphiphilic block copolymer or graft copolymer with relative high grafting degree of hydrophobic content, these poly( $\gamma$-glutamic acid)-graft-PLLA ( $\gamma$-PGA- $g$-PLA) copolymers have a very low grafting degree. Therefore, it is suggested that the core of the NPs is formed not only by PLA, but also by the main chain $\gamma$-PGA. Their hydrophilic domains are either exposed to the aqueous solvent or to a low level of hydrophilic domains present within the NPs. In this study, the 


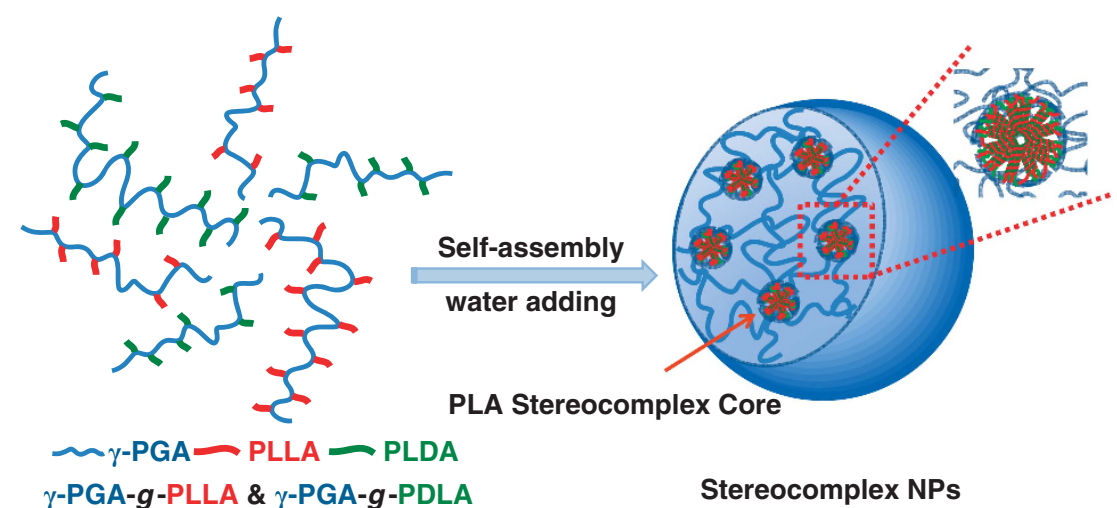

Scheme 2 Schematic illustration of nanoparticle formation by stereocomplexation of poly( $\gamma$-glutamic acid) ( $\gamma$-PGA)-g-PLLA/PDLA (poly(L-lactide)/poly(Dlactide)) copolymers.

Table 3 Characterization of NPs formed by individual and mixed $\gamma$-PGA-g-PLA copolymers

\begin{tabular}{|c|c|c|c|c|c|}
\hline \multirow[b]{2}{*}{ Sample } & \multicolumn{2}{|c|}{ NP size $(n m)$} & \multirow[b]{2}{*}{$\mu_{2} / \Gamma^{2 a}$} & \multirow{2}{*}{$\begin{array}{l}\text { Stereocomplex } \\
\text { NP size }(n m)\end{array}$} & \multirow[b]{2}{*}{$\mu_{2} / \Gamma^{2 \mathrm{a}}$} \\
\hline & Peak 1 & Peak 2 & & & \\
\hline$\gamma$-PGA-L 1 & $35 \pm 0.5$ & $240 \pm 4.6$ & 0.47 & $190 \pm 10.0$ & 0.30 \\
\hline$\gamma$-PGA-D 2 & $40 \pm 0.7$ & $250 \pm 5.5$ & 0.56 & & \\
\hline$\gamma-\mathrm{PGA}-\mathrm{L}_{3}$ & $110 \pm 2.1$ & - & 0.27 & $145 \pm 13.2$ & 0.22 \\
\hline$\gamma-\mathrm{PGA}^{-\mathrm{D}_{4}}$ & $140 \pm 2.8$ & - & 0.31 & & \\
\hline$\gamma-P G A-L_{5}$ & $140 \pm 4.6$ & - & 0.20 & $250 \pm 17.2$ & 0.18 \\
\hline$\gamma$-PGA-D 6 & $170 \pm 5.5$ & - & 0.30 & & \\
\hline
\end{tabular}

Abbreviations: NPs, nanoparticles; $\gamma$-PGA-g-PLA, poly( $\gamma$-glutamic acid)-graft-poly(L-lactide). apolydispersity factors.

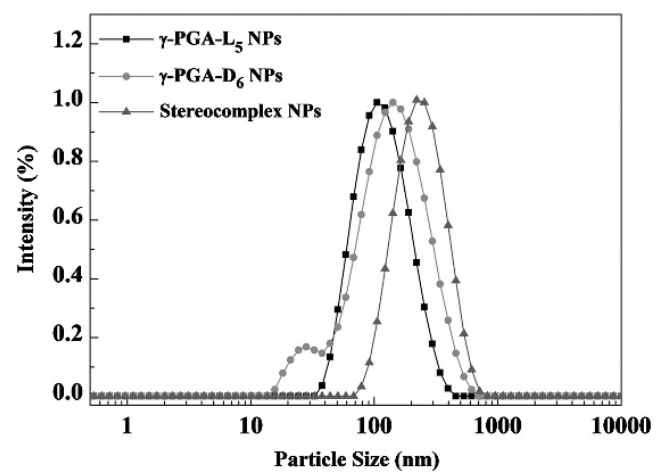

Figure 2 Size distribution of poly $\left(\gamma\right.$-glutamic acid) $(\gamma-P G A)-L_{5}$ nanoparticles (NPs), $\gamma$-PGA-D 6 NPs and $\gamma$-PGA-L $L_{5}+\gamma$-PGA-D 6 NPs in water at the concentration of $1 \mathrm{mg} \mathrm{ml}^{-1}$. A full color version of this figure is available at Polymer Journal online.

grafting degrees of PLA in graft copolymers were in the range from 0.2 to 1 per 100 glutamic acid units of $\gamma$-PGA. This means the large number of carboxyl groups of $\gamma$-PGA remain in/on the NPs.

\section{Wide-angle XRDmeasurement of $\gamma$-PGA- $g$-PLA NPs}

In order to further study the physical state of the NP core, XRD measurements were carried out on lyophilized NPs consisting of copolymers and a stereocomplex. Measurements on $\gamma$-PGA- $\mathrm{L}_{5}$, $\gamma$-PGA-D $D_{6}$, and stereocomplex of $\gamma$-PGA-L $L_{5}+\gamma$-PGA-D $D_{6}$ were carried out (Figure 5). There were no crystalline peaks in the case of $\gamma$-PGA- $\mathrm{L}_{5}$ and $\gamma$-PGA-D D $_{6}$ copolymer NPs, indicating that the core of NPs formed by copolymers only were amorphous. However, in the

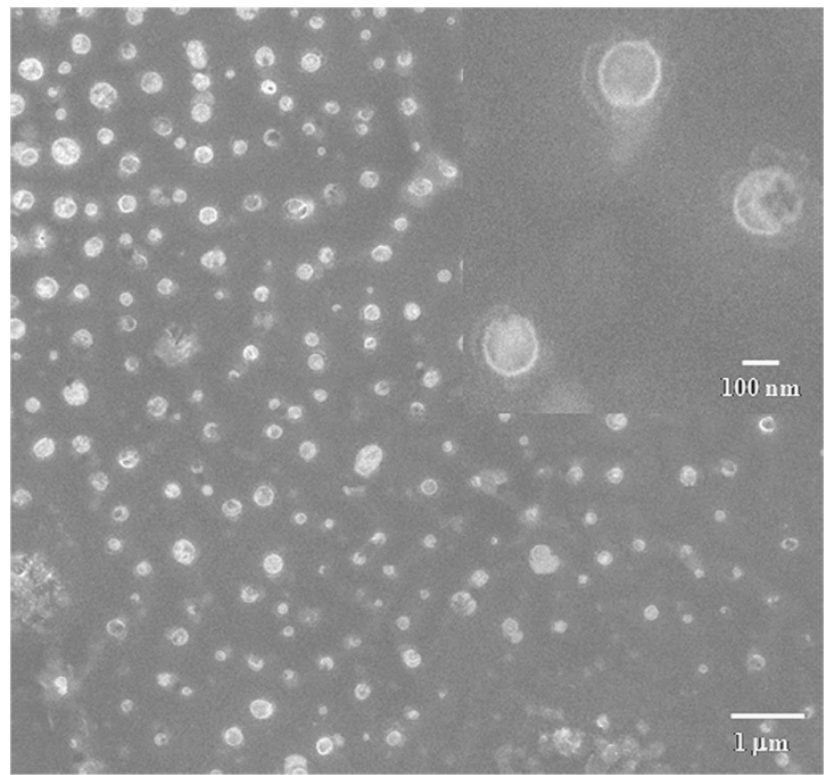

Figure 3 Scanning electron microscopy (SEM) images of poly( $\gamma$-glutamic acid) ( $\gamma$-PGA)-L $L_{5}+\gamma$-PGA-D 6 stereocomplex nanoparticles (NPs). A full color version of this figure is available at Polymer Journal online.

case of NPs formed by stereocomplex $\gamma$-PGA-L ${ }_{5}+\gamma-$ PGA-D $_{6}$, the crystalline peak at $2 \theta$ values of $12^{\circ}$ and $21^{\circ}$ were detected, which are characteristic peaks of the PLA stereocomplex ( $\beta$-form). Furthermore, the amount of PLA content in the copolymers had an important role on stereocomplex formation. With increasing PLA content in the copolymers, as compared with the $\gamma$-PGA- $\mathrm{L}_{3}+\gamma-\mathrm{PGA}_{-} \mathrm{D}_{4}$ stereocomplex NPs XRD patterns, the $\gamma$-PGA- $\mathrm{L}_{5}+\gamma$-PGA-D ${ }_{6}$ stereocomplex NPs yielded relatively clear crystalline peaks at $12^{\circ}$ and $21^{\circ}$, but at a very low intensity, which means that the majority part of the particle core was amorphous (Figure 5b).

\section{CONCLUSION}

In conclusion, the amphiphilic graft copolymers, $\gamma$-PGA-g-PLLA and $\gamma$-PGA- $g$-PDLA, consisting of a hydrophilic backbone of $\gamma$-PGA and hydrophobic side chains of enantiomeric PLLA or PDLA, were successfully prepared. The number of enantiomeric PLA chains coupled onto $\gamma$-PGA can be controlled by changing the molar amount of PLA added into the reaction system. These $\gamma$-PGA- $g$ PLA copolymers could form NPs, and stereocomplex crystallites were 


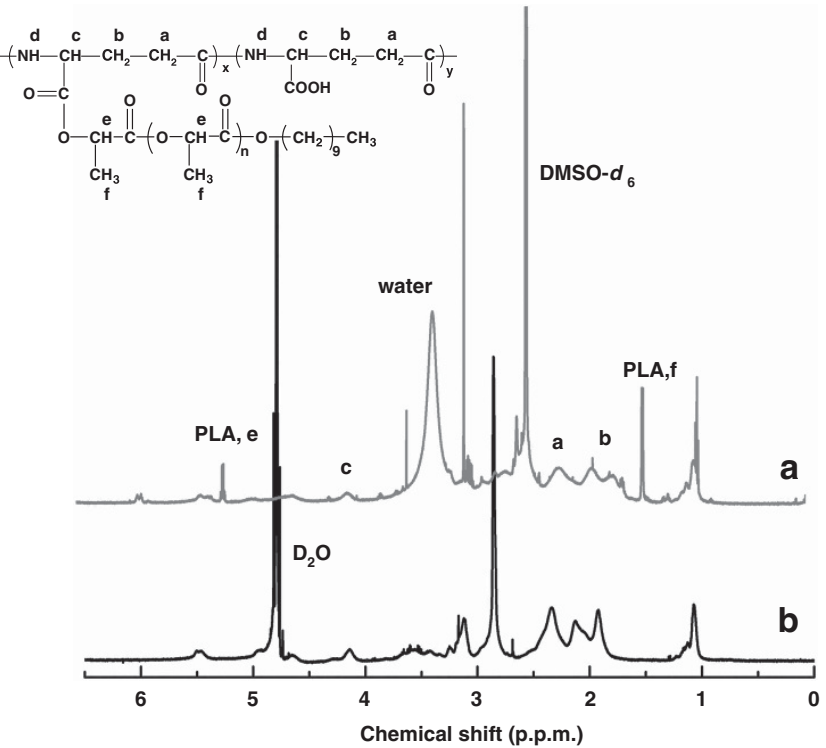

Figure $4{ }^{1} \mathrm{H}$ NMR spectra of (a) poly $\left(\gamma\right.$-glutamic acid) $\left(\gamma\right.$-PGA)- $\mathrm{L}_{3}$ in DMSO$d_{6}$ and (b) $\gamma-P G A-L_{3} N P$ dispersed in $D_{2} O$. A full color version of this figure is available at Polymer Journal online.
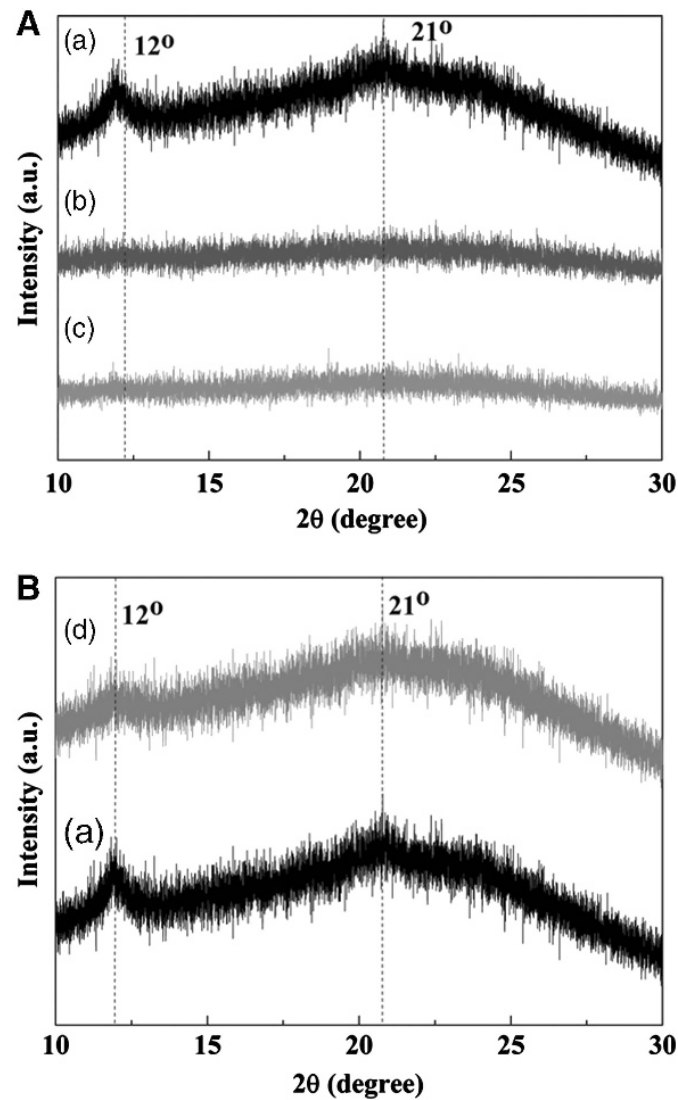

Figure 5 X-ray diffraction (XRD) patterns of nanoparticles (NPs) formed by (A) (a) stereocomplex of poly $(\gamma$-glutamic acid) $(\gamma$-PGA)-L5 $+\gamma$-PGA-D 6 copolymers, (b) $\gamma-P G A-L_{5}$ copolymer and (c) $\gamma-P G A-D_{6}$ copolymer; (B) (d) stereocomplex of $\gamma-\mathrm{PGA}-\mathrm{L}_{3}+\gamma-\mathrm{PGA}-\mathrm{D}_{4}$ copolymer. A full color version of this figure is available at Polymer Journal online. formed in the case of the mixture of $\gamma$-PGA- $g$-PLLA and $\gamma$-PGA- $g$ PDLA copolymers having a relatively large number of PLA grafts. It is expected that the stability and degradation behavior of these NPs can be regulated by controlling the crystallinity and stereocomplex formation of the core of these NPs. Moreover, the NPs consisting of $\gamma$-PGA- $g$-PLA have a large number of carboxyl groups on/into the NPs. Thus, these NPs should be potentially applicable for the immobilization of bioactive agents such as proteins, peptides and cationic molecules. Further research into their applications as drug delivery carriers are now in progress.

\section{ACKNOWLEDGEMENTS}

This work was supported by CREST from the Japan Science and Technology Agency (JST).

1 Zhang, L. \& Eisenberg, A. Multiple morphologies of 'crew-cut' aggregates of polystyrene-b-poly(acrylic acid) block copolymers. Science 268, 1728-1731 (1995).

2 Choucair, A. \& Eisenberg, A. Control of amphiphilic block copolymer morphologies using solution conditions. Eur. Phys. J. E Soft Matter 10, 37-44 (2003).

3 Cai, C., Lin, J., Chen, T. \& Tian, X. Aggregation behavior of graft copolymer with rigid backbone. Langmuir 26, 2791-2797 (2010).

4 Holder, S. J. \& Sommerdijk, M. New micellar morphologies from amphiphilic block copolymers: disks, toroids and bicontinuous micelles. Polym. Chem. 2, 1018-1028 (2011).

5 Gaucher, G., Dufresne, M. H., Sant, V. P., Kang, N., Maysinger, D. \& Leroux, J. C. Block copolymer micelles: preparation, characterization and application in drug delivery. J. Control. Release 109, 169-188 (2005).

6 Nishiyama, N. \& Kataoka, K. Nanostructured devices based on block copolymer assemblies for drug delivery: designing structures for enhanced drug function. Adv. Polym. Sci. 193, 67-101 (2006).

7 Letchford, K. \& Burt, H. A review of the formation and classification of amphiphilic block copolymer nanoparticulate structures: micelles, nanospheres, nanocapsules and polymersomes. Eur. J. Pharm. Biopharm. 65, 259-269 (2007).

8 Zhang, C., Qu, G., Sun, Y., Wu, X., Yao, Z., Guo, Q., Ding, Q., Yuan, S., Shen, Z., Ping Q. \& Zhou, H. Pharmacokinetics, biodistribution, efficacy and safety of N-octyl-Osulfate chitosan micelles loaded with paclitaxel. Biomaterials 29, 1233-1241 (2008).

9 Li, X., Qian, Y., Liu, T., Hu, X., Zhang, G., You, Y. \& Liu, S. Amphiphilic multiarm star block copolymer-based multifunctional unimolecular micelles for cancer targeted drug delivery and MR imaging. Biomaterials 32, 6595-6605 (2011).

10 Bae, Y. H. \& Yin, H. Stability issues of polymeric micelles. J. Control. Release 131, 2-4 (2008).

11 Chen, S., Cheng, S. \& Zhuo, R. Self-assembly strategy for the preparation of polymer-based nanoparticles for drug and gene delivery. Macromol. Biosci. 5, 576-589 (2011).

12 Schomaker, E. \& Challa, G. Complexation of stereoregular poly(methyl methacrylates). 14. The basic structure of the stereocomplex of isotactic and syndiotactic poly(methyl methacrylate). Macromolecules 22, 3337-3341 (1989).

13 Tsuji, H. Poly(lactide) Stereocomplexes: formation, structure, properties, degradation, and applications. Macromol Biosci. 5, 569-597 (2005).

14 Fukushima, K., Pratt, R., Nederberg, F., Tan, J., Yang, Y., Waymouth, R. \& Hedrick, J. Organocatalytic approach to amphiphilic comb-block copolymers capable of stereocomplexation and self-assembly. Biomacromolecules 9, 3051-3056 (2008).

$15 \mathrm{Kim}, \mathrm{S} . \mathrm{H}$., Tan, J. P. K., Nederberg, F., Fukushima, K., Yang, Y, Y., Waymouth, R. M. \& Hedrick, J. L. Mixed micelle formation through stereocomplexation between enantiomeric poly(lactide) block copolymers. Macromolecules 42, 25-29 (2009).

16 Tan, J. P. K., Kim, S. H., Nederberg, F., Apple, E. A., Waymouth, R. M., Zhang, Y. Hedrick, J. L. \& Yang, Y. Y. Hierarchical supermolecular structures for sustained drug release. Small 5, 1504-1507 (2009).

17 Chen, L., Xie, Z., Hu, J., Chen, X. \& Jiang, X. Enantiomeric PLA-PEG block copolymers and their stereocomplex micelles used as rifampin delivery. J. Nanoparticle Res. 9, 777-785 (2007)

18 Nederberg, F., Apple, E., Tan, J. P., Kim, S. H., Fukushima, K., Sly, J., Miller, R. Waymouth, R., Yang, Y. Y. \& Hedrick, J. L. Simple approach to stabilized micelles employing miktoarm terpolymers and stereocomplexes with application in paclitaxel delivery. Biomacromolecules 10, 1408-1460 (2009).

19 Kang, N., Perron, M. E., Prud'homme, R. E., Zhang, Y., Gaucher, G. \& Leroux, J. C. Stereocomplex block copolymer micelles: core-shell nanostructures with enhanced stability. Nano Lett. 5, 315-319 (2005)

20 Nagahama, K., Mori, Y., Ohya, Y. \& Ouchi, T. Biodegradable nanogel formation of polylactide-grafted dextran copolymer in dilute aqueous solution and enhancement of its stability by stereocomplexation. Biomacromolecules 8, 2135-2141 (2007).

21 Kim, T. W., Lee, T. Y., Bae, H. C., Hahm, J. H., Kim, Y. H., Park, C., Kang, T. H., Kim, C. J., Sung, M. H. \& Poo, H. Antitumor immunity poly- $\gamma$-glutamate induces NK cellmediated oral administration of high molecular mass. J. Immunol. 179, 775-780 (2007). 
22 Uto, T., Akagi, T., Yoshinaga, K., Toyama, M., Akashi, M. \& Baba, M. The induction of innate and adaptive immunity by biodegradable poly $(\gamma$-glutamic acid) nanoparticles via a TLR4 and MyD88 signaling pathway. Biomaterials 32, 5206-5212 (2011).

23 Matsusaki, M., Hiwatari, K., Higashi, M., Kaneko, T. \& Akashi, M. Stably-dispersed and surface-functional bionanoparticles prepared by self-assembling amphipathic polymers of hydrophilic poly(g-glutamic acid) bearing hydrophobic amino acids. Chem. Lett. 33 398-399 (2004).

24 Akagi, T., Kaneko, T., Kida, T. \& Akashi, M. Preparation and characterization of biodegradable nanoparticles based on poly ( $\gamma$-glutamic acid) with L-phenylalanine as a protein carrier. J. Control Release 108, 226-236 (2005).

25 Akagi, T., Wang, X., Uto, T., Baba, M. \& Akashi, M. Protein direct delivery to dendritic cells using nanoparticles based on amphiphilic poly(amino acid) derivatives. Biomaterials 28, 3427-3436 (2007).
26 Akagi, T., Baba, M. \& Akashi, M. Preparation of nanoparticles by the self-organization of polymers consisting of hydrophobic and hydrophilic segments: potential applications. Polymer 48, 6729-6747 (2007).

$27 \mathrm{Kim}, \mathrm{H}$., Akagi, T. \& Akashi, M. Preparation of size tunable amphiphilic poly(amino acid) nanoparticles. Macromol. Biosci. 9, 825-932 (2009).

28 Akagi, T., Watanabe, K., Kim, H. \& Akashi, M. Stabilization of polyion complex nanoparticles composed of poly(amino acid) using hydrophobic interactions. Langmuir 26, 2406-2413 (2010).

29 Akagi, T., Kaneko, T., Kida, T. \& Akashi, M. Multifunctional conjugation of proteins on/ into bio-nanoparticles prepared by amphiphilic poly( $\gamma$-glutamic acid). J. Biomater. Sci. Polym. Ed. 17, 875-892 (2006).

30 Desbaumes, L. \& Eisenberg, A. Single-solvent preparation of crew-cut aggregates of various morphologies from an amphiphilic diblock copolymer. Langmuir 15, 36-38 (1999). 